\title{
The changing investment in translational science by academic medical centers: HOPE in the Valley of Death
}

\author{
Lucy Y. Ghoda, ${ }^{1,2}$ Steven T. Rosen, ${ }^{1,3,4}$ and Larry W. Kwak ${ }^{1,3,4}$ \\ 'Comprehensive Cancer Center and Beckman Research Institute, ${ }^{2}$ Cehr Family Center for Leukemia Research, ${ }^{3}$ Toni Stephenson Lymphoma Center, and ${ }^{4}$ Department of Hematology and Hematopoietic Stem \\ Cell Transplantation, City of Hope, Duarte, California, USA.
}

$\mathbf{H}$ istorically, academic medical centers (AMCs) have been pioneers in medicine with a mission to advance promising discovery research to clinical application. Moreover, an institution's prestige has been based on the reputation, innovation, and deep research and clinical expertise of its faculty. A large portion of basic research has been funded through federal agencies (e.g., NIH). An added bonus is that some research has yielded patentable inventions that, if successfully commercialized, became a revenue stream for the institution. Nevertheless, most investigators have not actively pursued commercialization of discoveries, leaving those responsibilities to their technology licensing offices.

Now, a shifting technologic and economic landscape is transforming the way research is conducted in AMCs. One major factor is the de-emphasis of preclinical research by major biotech and pharma. Increasingly, early development of new therapeutics is the product of AMCs or small start-up companies. In some cases, intellectual property (IP) is licensed to start-ups whose sole focus is the development and commercialization of that IP. Only when early development efforts show promise does a major company step in with a collaboration, alliance, or acquisition. Thus, there is more investment by venture funds to promote early-stage discoveries. At the same time, many academic investigators have shifted their research focus from basic to translational science, energized by seeing their or their colleague's work enter into clinical trial and, in some cases, become an approved product. For the institution, translational research is more costly, but can be more attractive to industry, leading to academia-industry alliances and shifting the composition of funding sources. In turn, commercial funding can support important translational research less likely to be funded by conventional peer-reviewed grants. Finally, philanthropic organizations often require specific deliverables needed for commercializing a discovery, e.g., the completion of toxicology studies or the filing of an investigational new drug (IND), in return for continued investment. Some have even required a contractual agreement to share revenues that result from IP licensing as a condition of funding.

To adapt and compete in this new environment, many AMCs have changed the way they manage research discoveries. Whereas in the past licensing was a serendipitous or passive occurrence, technology licensing offices of today actively pursue biotech, big pharma, and venture funds for licensing. Upstream of licensing, investigators are becoming more sophisticated in their understanding of product commercialization and institutions are financing innovative research with potential for licensing. To support these endeavors, infrastructure investments are being made to attract more research funding and more translational researchers. Thus, AMCs with entrepreneurial investigators together with a strong infrastructure for translational research are bound to be more successful in capturing robust funding. Accord-

Conflict of interest: STR is on the speaker's bureau for Celgene, Global Education Group, Paradign Medical Communications, LLC, and Abbvie; consults for Novartis Pharmaceuticals Corporation, Pepromene Bio, Inc., Exicure, and Apobiologix/Apotex, Inc.; serves on the external advisory boards of Seattle Genetics, NeoGenomics, and Aileron Therapeutics, Inc.; and holds stock options in Pepromene Bio, Inc., and Exicure. LWK consults for Celltrion, CJ Healthcare, Enzychem LifeSciences, InnoLifes, Kolon Life Sciences, and Pepromene Bio; serves on advisory boards of Genentech and Teva; has equity ownership in Agenus, InnoLifes, Pepromene Bio, and Xeme BioPharma, Inc.; and receives research funding from Pepromene Bio.

Copyright: () 2020, American Society for Clinical Investigation.

Reference information: / Clin Invest. 2020;130(7):3333-3335. https://doi.org/10.1172/JCI138640.

ingly, creating the optimal environment for such research may logically become a priority (1).

\section{Desirable characteristics of a translational accelerator}

Judicious allocation of resources for support of pipeline products could potentially accelerate licensing and facilitate successful regulatory approval. Funding the late preclinical development phase, i.e., after a lead product candidate is identified, is a particularly attractive opportunity, as it is underfunded by the NIH. In fact, this phase of product development was recognized by the former NIH director, Elias Zerhouni, as the "Valley of Death." Promising ideas often fail because commercially directed research is significantly more expensive than early-stage discovery, as it can involve lengthy process development, scale-up, and IND-enabling toxicology testing. Support of this phase, however, may have significant financial impact for the institution, starting with upfront and milestone licensing payments, which can align the risk/reward ratio between AMCs and licensees, and can serve to incentivize faculty by elevating an inventor's status among peers. After approval, downstream royalties from an institution's product can be transformative.

Some crucial characteristics of programs structured to support late preclinical product development are in Table 1. Potentially underappreciated is the perception of equity of access, which is required for a collaborative, yet competitive, research environment. It is important that the application process be advertised throughout the institution. Reviews should be conducted by specific experts devoid of conflicts of interest, and outcomes should align with publicized criteria. Other key operational considerations include the following:

Fund eligibility. Limiting eligibility to late preclinical development potentially prevents competition in areas where exter- 


\section{Table 1. Ideal components of a successful academic translational accelerator funding program}

Characteristics

Viewed as fair and equitable by the institution's
investigators

Integrated and synergistic with other mechanisms of support for superior science such as grants, contracts, and philanthropic contributions; the program should not be perceived as, or does not play the role of, a life raft for unfunded or inferior science

Serves to stimulate or enhance good science and motivate investigators to expand their horizon into the translational arena

Does not have an unduly burdensome application and reporting process

Has clear and objective metrics for success that are articulated at the time of funding approval Has an adequate set of support systems to achieve success

Is cost effective while maintaining realistic short-term expectations for success from the institutional point of view

\section{Operational practices to support the program}

- Formal application process

- Opportunity widely advertised across the institution

- Funding decisions are consistent with the criteria published in the request for applications

- Investigators funded through the program should be in good standing, having a record of extramural funding, publications, and other performance standards of the institution

- Awards should serve to create new knowledge otherwise not attainable through conventional funding mechanisms

- Provide opportunities to mentor investigators not familiar with translating bench science to commercial opportunities

- Design templates to solicit application-specific information

- Minimal requirement for free form-written material

- Milestone-driven projects

- Operational "Co/No Go" milestones to minimize funding of futile projects

- Institutional infrastructure should exist to support multiple projects heading to commercialization

- Provide project management and guidance to minimize efforts expended by the investigator to reinvent the wheel

- Pay out funds by milestone achievement with clear Co/No Go criteria

- Refrain from attempting to achieve unrealistic returns on investment (ROIs) early in the development cycle

- Focus efforts on licensing to maximize future ROIs rather than short-term licensing fees nal funding is available. Project readiness can be defined by availability of in vivo proof-of-concept efficacy, preceded by strong evidence for target validation. In addition, robust physicochemical data (e.g., co-crystallization) in support of drug-target interactions should be encouraged. Other measures of product readiness are proximity to pre-IND filing, including lead candidate characterization, preliminary toxicology, and clinical manufacturing and clinical trial concepts. Finally, IP protection for the product is critically important for licensing. Adherence to these requirements facilitates triage of immature projects or those lacking commercialization potential.

Application review. An efficient application process minimizes unnecessary written material that is both time consuming for applicants and difficult for reviewers to digest. Short templated project abstracts can be used for prescreening to triage projects that do not fulfill basic eligibility requirements. In preparing full applications, an iterative process can provide opportunities to coach applicants without experience in translational product development. For example, opportunities for applicants to present their project to a committee of experienced reviewers can provide valuable positive feedback, and gaps can be identified. Finally, an objective scoring system can be useful for comparative analyses of characteristics of projects accepted (or not) and subsequent achievement of milestones.

Program management. Incremental milestone funding decreases the investment risk while simultaneously enabling better monitoring of progress toward IND filing. To eliminate ambiguities, the plan itself should be structured in a way that achievement of a milestone can be easily determined. An experienced project manager can be central to assessing milestone achievement, anticipating and resolving bottlenecks, assisting with study reports, educating faculty on regulatory and manufacturing processes, and serving as liaison to contract research organizations (CROs).

Facilities to support the translational accelerator. The optimal level of infrastructure necessary to leverage commercialization opportunities depends greatly on the size and research output of the institution (2). However, this capability should include in-house GMP-compliant manufacturing, manufacturing process development, project management, and business development. A major investment in GMP manufacturing will also require qualityassurance capability for managing product release, facilities operations, and auditing activities. Finally, a regulatory capability, which oversees FDA submissions and compliance with other regulatory agencies, is critical for an efficient pipeline to the clinic. The regulatory group must also work with quality assurance and statisticians to ensure data integrity for regulatory document submissions. GMP manufacturing capabilities can also potentially decrease project risk and thereby be leveraged to enhance licensing terms.

\section{The HOPE Portfolio Fund}

One academic model of a translational accelerator, the HOPE Portfolio Fund, was created in 2013 at City of Hope (COH). External advisors from venture capital, biotech, and pharma sectors were recruited to bring objectivity to the review process and to share knowledge of the requirements for market success, attractiveness to industry, and technology conversion. The program accepts projects in late preclinical development, typically after the target has been validated, a lead identified, and proof of concept demonstrated in animal models. The institution must have an assigned IP interest. Discovery-phase research is specifically not supported. Preapplications are screened for eligibility and those that meet published criteria are invited to submit a full application, including in-person 
presentations to an internal committee prior to formal review by external advisors.

Two successful examples of the program are $\mathrm{COH} 29$, an orally administered small-molecule inhibitor of ribonucleotide reductase $(2,3)$, and a monoclonal antibody $(\mathrm{mAb})$ targeting $\mathrm{B}$ cell-activating factor receptor (BAFF-R) intended for the treatment of $\mathrm{B}$ cell malignancies (4, 5 ), both discovered at $\mathrm{COH}$. The program funded toxicology studies of $\mathrm{COH} 29$, cGMP manufacturing, and a phase I clinical trial at $\mathrm{COH}$. It was licensed to a biotech company just before clinical trial activation. For the BAFF-R mAb, program funding was used to humanize and optimize the lead at a CRO and conduct INDenabling toxicology studies, activities often outside of the scope of academia. It was licensed earlier than anticipated, before completion of these milestones, due to intense commercial interest, and a sponsored first-in-human clinical trial is anticipated to begin in 2020.

Program investments to date total \$7.7 million (\$7.7 M; with commitments of $\$ 17.7 \mathrm{M}$ for all milestones). Five licenses and/or options have been negotiated from four awarded projects resulting in $\$ 14.1 \mathrm{M}$ income from licensing agreements, which includes upfront, annual milestone payments and sponsored research agreements. Thus, short-term returns are promising. Parenthetically, these figures represent only a fraction of the total investment in research by the institution, including a more sizeable investment in discovery research.

\section{Concluding remarks}

Novel medical therapeutic development is at risk of languishing in the Valley of Death, often because of insufficient access to suitable funding or lack of expertise to develop ideas beyond the academic laboratory. A formal program with transparent processes to fund new product development at the late preclinical stage can serve to accelerate commercialization of academic research. When such a program is supported by institutional manufacturing and regulatory infrastructure and input from product development content experts, the likelihood of reaching clinical stage evaluation is enhanced. Such efforts have the potential to extend patent life, enhance commercial interest, and result in more lucrative licensing agreements in the near term. Should the product advance further commercially and receive regulatory approval, the long-term benefits to the institution and inventors through royalties can be substantial.

Because not all ideas or inventions can be expected to generate a return, establishing a system to carefully evaluate opportunities to advance products to firstin-human studies can make a difference. Ultimately, bringing discoveries to market and thereby improving the health of patients is the inspiration for accelerating translational research by AMCs.

\section{Acknowledgments}

The authors would like to thank Robert Stone for ongoing support of the HOPE Portfolio Fund and its external advisors for sharing their experiences and expertise in the areas of biotechnology, pharma, and venture capital funding. The authors would also like to acknowledge the assistance of Giles and Associates, City of Hope colleagues, George Megaw, Ashley Lee, Harlan Levine, John Zaia,
Michael Friedman, David Horne, Linda Malkas, Christoph Pittius, and Lynne Smith for editorial comments and suggestions. We are grateful for generous support from the National Cancer Institute (SPORE 2P50CA107399 to LWK) and Leukemia and Lymphoma Society (MCL SCOR 7000-18 to LWK). Research projects in the HOPE Portfolio Fund reported in this publication included work performed in the Chemical GMP Synthesis Manufacturing Facility and the Hematopoietic Tissue Biorepository (Pathology) Cores supported by the National Cancer Institute of the NIH under grant number P30CA033572. The content is solely the responsibility of the authors and does not necessarily represent the official views of the NIH.

Address correspondence to: Larry W. Kwak, Beckman Research Institute, Room 4117, City of Hope, 1500 East Duarte Road, Duarte California 910103000, USA. Phone: 626.218.0025; Email: lkwak@coh.org.

1. Grunseth M, Chen C, Kelly R, Cook SB, Megaw $\mathrm{G}$, Couture L. Surveying the best in translation. Nat Biotechnol. 2014;32(5):415-418.

2. Zhou B, et al. A small-molecule blocking ribonucleotide reductase holoenzyme formation inhibits cancer cell growth and overcomes drug resistance. Cancer Res. 2013;73(21):6484-6493.

3. Chen MC, et al. The novel ribonucleotide reductase inhibitor COH29 inhibits DNA repair in vitro. Mol Pharmacol. 2015;87(6):996-1005.

4. Qin H, et al. Novel BAFF-receptor antibody to natively folded recombinant protein eliminates drug-resistant human b-cell malignancies in vivo. Clin Cancer Res. 2018;24(5):1114-1123.

5. Qin H, et al. CAR T cells targeting BAFF-R can overcome CD19 antigen loss in B cell malignancies. Sci Transl Med. 2019;11(511):eaaw9414. 